\title{
REMARK ON A PAPER BY ACZÉL AND OSTROWSKI
}

\author{
WOLFGANG WALTER
}

(Received 29 March 1974)

Communicated by J. B. Miller

The objective of the paper Aczél and Ostrowski (1973) is to show in an :lementary way that any real-valued function $f$, defined on $(0,1)$ and satisfying he inequality

$$
\sum_{k=1}^{n} p_{k} f\left(q_{k}\right) \leqq \sum_{k=1}^{n} p_{k} f\left(p_{k}\right) \text { for } p_{k}, q_{k}>0, \sum_{k=1}^{n} p_{k}=\sum_{k=1}^{n} q_{k}=1,
$$

where $n \geqq 3$ is fixed, is necessarily of the form

$$
f(p)=a \log p+b \text { for } p \in(0,1), \text { where } a \geqq 0 .
$$

The proof given in Aczél and Ostrowski (1973) is split up in two steps, (i) $f$ is weakly) increasing, (ii) $p D f(p)=$ const. $\geqq 0$ in $(0,1)$, where $D f$ is the derivative or a fixed Dini derivative of $f$. For the second step, two different proofs are given n Aczél and Ostrowski (1973). The first proof uses the fact that a monotonic unction is almost everywhere differentiable, while the second proof uses two :lementary theorems on Dini derivatives, one of them being Scheeffer's heorem.

In what follows, another proof of statement (ii) is given which does not use iny results about Dini derivatives (though the notion of a Dini derivative is Ised). The proof uses the following arithmetic mean property $(M)$ of divided lifferences.

(M) Let $s_{k}=s+k h(k=1, \cdots, n)$, where $s$ and $h>0$ are given. Then the livided difference (of any function) over the interval $\left(s, s_{n}\right)$ is equal to the Irithmetic mean of the divided differences over the intervals $\left(s, s_{1}\right),\left(s_{1}, s_{2}\right), \cdots$, $\left.s_{n-1}, s_{n}\right)$.

The starting point for the proof are the two inequalities (13) and (15) of tczél and Ostrowski (1973) which are given here in a slightly different notation:

$$
p \frac{f(p+h)-f(p)}{h} \leqq r \frac{f(r)-f(r-h)}{h} \text { for } 0<h<r, p+r<1
$$


(3)

$(r-h) \frac{f(r)-f(r-h)}{h} \leqq(p+h) \frac{f(p+h)-f(p)}{h}$ for $0<h<r, p+r<1$.

Let $0<h<s, s_{n}=s+h n$. Then

$$
\frac{p}{p+h} s \frac{f\left(s_{n}\right)-f(s)}{s_{n}-s} \leqq p \frac{f(p+h)-f(p)}{h} \leqq s_{n} \frac{f\left(s_{n}-f(s)\right.}{s_{n}-s} \text { for } p+s_{n}<1 .
$$

The inequality at right follows from (2), applied to $r=s_{1}, \cdots, s_{n}$ and $(M)$, the inequality at left follows from (3) in the same way. All further considerations are based on (4).

Let $s$ be a small positive number, let $s \leqq p \leqq 1-4 s$ and let, for a given $h<s, s_{n}$ be chosen in such a way that $2 s<s_{n} \leqq 3 s$. Then it follows from (4) that

$$
\frac{f(p+h)-f(p)}{h} \leqq C(s)=\frac{3}{s}(f(3 s)-f(s)) \text { for } s \leqq p \leqq 1-4 s .
$$

Here we have used the fact that $f$ is increasing. Using $(M)$, it follows that any divided difference over a subinterval of $[s, 1-4 s]$ is bounded above by $C(s)$. Hence, $f$ is locally Lipschitz continuous in $(0,1)$, and all Dini derivatives are finite.

Next, we fix $t>s$, let $h \rightarrow 0$ and choose $n=n(h)$ in such a way that $s_{n} \rightarrow t$ as $h \rightarrow 0$. Then we obtain from (4)

$$
s \frac{f(t)-f(s)}{t-s} \leqq p D_{+} f(p) \leqq p D^{+} f(p) \leqq t \frac{f(t)-f(s)}{t-s} \text { for } p+t<1 .
$$

Letting $s \rightarrow t-0$, it follows that

$$
t D^{-} f(t) \leqq p D_{+} f(p) \leqq p D^{+} f(p) \leqq t D_{-} f(t) \text { for } p+t<1 .
$$

These inequalities imply that $f^{\prime}(p)$ exists and $p f^{\prime}(p)=$ const. $\geqq 0$ in $(0,1)$.

\section{Reference}

J. Aczél and A. M. Ostrowski (1973), 'On the characterization of Shannon's entropy by Shannon's inequality', J. Austral. Math. Soc. 16, 368-374.

Professor Dr. Wolfgang Walter

Mathematisches Institut I

Universität Karlsruhe

75 Karlsruhe, Germany.

University of Waterloo

Waterloo, Ontario

Canada. 